\title{
Spin-orbit torques in a Rashba honeycomb antiferromagnet
}

\author{
Robert Sokolewicz $\odot,{ }^{1}$ Sumit Ghosh $\odot,{ }^{2,3}$ Dmitry Yudin, ${ }^{4,5}$ Aurélien Manchon, ${ }^{2}$ and Mikhail Titov ${ }^{1,6}$ \\ ${ }^{1}$ Institute for Molecules and Materials, Radboud University Nijmegen, NL-6525 AJ Nijmegen, The Netherlands \\ ${ }^{2}$ Physical Science and Engineering Division, King Abdullah University of Science and Technology, Thuwal 23955, Saudi Arabia \\ ${ }^{3}$ Peter Grünberg Institut, Forschungszentrum Jülich, 52425 Jülich, Germany \\ ${ }^{4}$ Skolkovo Institute of Science and Technology, Moscow 121205, Russia \\ ${ }^{5}$ Department of Physics and Astronomy, Uppsala University, Box 516, SE-751 20, Uppsala, Sweden \\ ${ }^{6}$ Department of Nanophotonics and Metamaterials, ITMO University, Saint Petersburg 197101, Russia
}

(Received 29 August 2019; revised manuscript received 18 November 2019; published 2 December 2019)

\begin{abstract}
Recent experiments on switching antiferromagnetic domains by electric current pulses have attracted a lot of attention to spin-orbit torques in antiferromagnets. In this work, we employ the tight-binding model solver, kwant, to compute spin-orbit torques in a two-dimensional antiferromagnet on a honeycomb lattice with strong spin-orbit interaction of Rashba type. Our model combines spin-orbit interaction, local $s$ - $d$-like exchange, and scattering of conduction electrons on on-site disorder potential to provide a microscopic mechanism for angular-momentum relaxation. We consider two versions of the model: One with preserved and one with broken sublattice symmetry. A nonequilibrium staggered polarization that is responsible for the so-called Néel spin-orbit torque is shown to vanish identically in the symmetric model but may become finite if sublattice symmetry is broken. Similarly, antidamping spin-orbit torques vanish in the symmetric model but become finite and anisotropic in a model with broken sublattice symmetry. As expected, antidamping torques also reveal a sizable dependence on impurity concentration. Our numerical analysis also confirms symmetry classification of spin-orbit torques and strong torque anisotropy due to in-plane confinement of electron momenta.
\end{abstract}

DOI: 10.1103/PhysRevB.100.214403

\section{INTRODUCTION}

Ferromagnetic spintronics has greatly contributed to microelectronic technology in the past few decades [1-3]. One of the practical results was the development of magnetic memories with purely electronic write-in and read-out processes as an alternative to existing solid state drive technologies $[4,5]$. An increasing demand for ever-higher performance computation and ever faster big data analytics has sparked recently the interest to antiferromagnetic spintronics [6-12], i.e., to the usage of much more subtle antiferromagnetic order parameter to store and process information. This idea is driven primarily by the expectation that antiferromagnetic materials may naturally allow for up to $\mathrm{THz}$ operation frequencies $[11,13,14]$ in sharp contrast to ferromagnets whose currentinduced magnetization dynamics is fundamentally limited to the $\mathrm{GHz}$ frequency range.

The best efficiency in electric switching of magnetic domains is achieved in systems involving materials with at least partial spin-momentum locking [15] due to sufficiently strong spin-orbit interaction. The latter is responsible for the so-called spin-orbit torques on magnetization that are caused by sizable nonequilibrium spin polarization induced by an electron flow [16-30].

Recently, spin-orbit-torque-driven electric switching of the Néel vector orientation has been predicted [18] and discovered in noncentrosymmetric crystals such as CuMnAs [8,15,22,31] and $\mathrm{Mn}_{2} \mathrm{Au}$ [32-34]. Even though many antiferromagnetic compounds are electric insulators [35], which limits the range of their potential applications, e.g., for spin injection [36], the materials like CuMnAs and $\mathrm{Mn}_{2} \mathrm{Au}$ possess semimetal and metal properties, inheriting strong spin-orbit coupling and sufficiently high conductivity. These materials also give rise to collective mode excitations in the $\mathrm{THz}$ range [34].

Spin-orbit torque in antiferromagnets has been investigated theoretically using the Kubo-Streda formula in the case of two-dimensional (2D) Rashba gas, as well as in tight-binding models of $\mathrm{Mn}_{2} \mathrm{Au}[18,37]$. These pioneering works describe the spin-orbit torques based on their influence on the currentdriven dynamics as predicted by the Landau-Lifshitz-Gilbert equation. In particular, they proposed that only a staggered fieldlike torque and a nonstaggered antidamping torque can trigger current-driven antiferromagnetic $\mathrm{THz}$ switching and excitation in antiferromagnets (see, e.g., Refs. [38-40]). This analysis served as a basis to further investigation of spinorbit torques in heterostructures [20,41,42]. Furthermore, a symmetry group analysis has been developed to predict the form of these two components based on the magnetic point groups of the antiferromagnets $[37,43]$.

In the context of current-driven antiferromagnetic domain wall [44] and skyrmion motion [45,46], the Néel vector dynamics has been modeled within the phenomenological treatment of the Landau-Lifshitz-Gilbert equation pioneered by Slonczewski [47]. In this phenomenological approach the torques on magnetization derived above have been simply postulated [13,48-50].

While most of the above studies have focused on the existence and influence of the staggered fieldlike and nonstaggered antidamping torques, in the present work, we compute all types of spin-orbit torques from a long-scale 
numerical analysis in a diffusive transport regime. Our study of spin-orbit torques is based on the numerical computation of nonequilibrium spin polarizations for an effective $s-d$ type model in a 2D honeycomb antiferromagnet with Rashba spin-orbit coupling and on-site disorder potential. Our results stress the importance of anisotropy of both fieldlike and antidamping like spin-orbit torques due to $2 \mathrm{D}$ confinement of conduction electrons. We also find, surprisingly, that both the staggered fieldlike and nonstaggered antidamping torques vanish identically in a model with an $s$ - $d$ exchange coupling that is the same on two antiferromagnetic sublattices. In contrast, the model with strongly asymmetric $s-d$ exchange couplings leads to finite antidamping torques while the entire notion of staggered torques becomes largely irrelevant in such an asymmetric model.

\section{MODEL}

In this paper, we develop a numerical framework for the microscopic analysis of spin-orbit torques that is realized with the help of the tight-binding model solver, kwant [51]. Our methodology is illustrated using an $s$ - $d$-type model for a 2D antiferromagnet on the honeycomb lattice depicted in Fig. 1(a). To be more specific, we consider the Kane-Mele tight-binding model for conduction electrons $[52,53]$ with the Hamiltonian

$$
H_{0}=H_{\mathrm{tb}}+H_{R}+H_{\mathrm{sd}},
$$

where the first term describes the nearest-neighbor hopping,

$$
H_{\mathrm{tb}}=-w \sum_{\langle i, j\rangle} \sum_{\sigma} c_{i \sigma}^{\dagger} c_{j \sigma}
$$

with the hopping energy $w$. The operators $c_{i \sigma}^{\dagger}\left(c_{i \sigma}\right)$ are the standard creation (annihilation) operators for a fermion on the lattice site $i$ with the spin index $\sigma$.

The term $H_{R}$ describes spin-orbit interaction of Rashba type that is responsible for spin-orbit torque on magnetization.
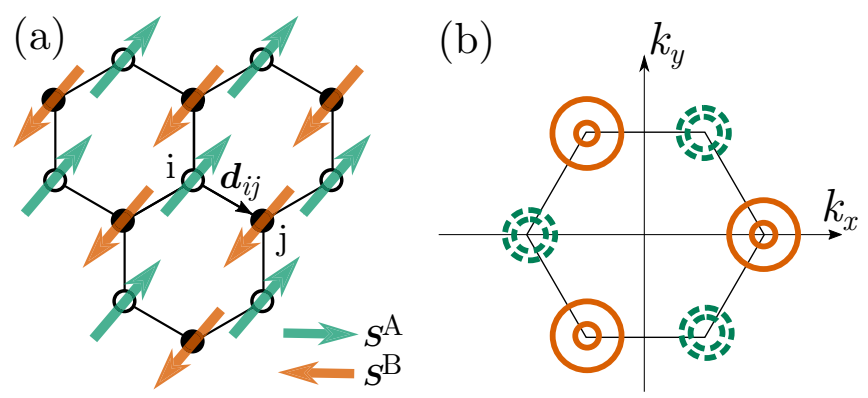

FIG. 1. (a) A two-dimensional honeycomb lattice hosting antiparallel localized magnetic moments $\boldsymbol{S}^{A}$ and $\boldsymbol{S}^{B}$ that induce opposite exchange potentials on A and B sublattices. The vector $\boldsymbol{d}_{i j}$ is directed from an A site $i$ to one of the three nearest-neighbor B sites $j$. (b) Fermi surfaces (strongly enlarged) in quasimomentum space for the Fermi energy $E=0.3 w$, couplings $\lambda=0.05 w, \Delta=J S=$ $0.1 w$, and $\theta=\pi / 2$. Solid (orange) and dashed (green) lines indicate $K$ and $K^{\prime}$ valleys.
This spin-orbit interaction is represented by the term

$$
H_{R}=\frac{i \lambda}{a} \sum_{\langle i, j\rangle} \sum_{\sigma \sigma^{\prime}} \hat{z} \cdot\left(\boldsymbol{\sigma} \times \boldsymbol{d}_{i j}\right)_{\sigma \sigma^{\prime}} c_{i \sigma}^{\dagger} c_{j \sigma^{\prime}},
$$

with the unit vector $\hat{z}$ directed perpendicular to the $2 \mathrm{D}$ crystal plane. The notation $\sigma=\left(\sigma_{x}, \sigma_{y}, \sigma_{z}\right)$ represents the threedimensional vector of Pauli matrices, the vectors $\boldsymbol{d}_{i j}$ connect neighboring sites as shown in Fig. 1(a), and $\lambda$ is the Rashbaspin-orbit strength. For any site $i$ on the sublattice $A$ we have three such vectors:

$$
\boldsymbol{d}_{1}=a\left(\begin{array}{l}
0 \\
1
\end{array}\right), \quad \boldsymbol{d}_{2}=\frac{a}{2}\left(\begin{array}{c}
\sqrt{3} \\
-1
\end{array}\right), \quad \boldsymbol{d}_{3}=-\frac{a}{2}\left(\begin{array}{c}
\sqrt{3} \\
1
\end{array}\right),
$$

where $a$ is the length of the bond between $A$ and $B$.

Finally, the $s$ - $d$-like exchange interaction between localized magnetic moments $\boldsymbol{S}_{i}$ and spins of conduction electrons is described by the term

$$
H_{\mathrm{sd}}=-J \sum_{i} \sum_{\sigma \sigma^{\prime}} \boldsymbol{S}_{i} \cdot \boldsymbol{\sigma}_{\sigma \sigma^{\prime}} c_{i \sigma}^{\dagger} c_{i \sigma^{\prime}},
$$

with the coupling strength $J$ taken here to be the same on $A$ and $B$ sublattices. The term (5) couples the tight-binding model for conduction electrons to a classical Heisenberg model for localized angular momenta $\boldsymbol{S}_{i}$ with antiferromagnetic ground state. The localized momenta $S_{i}$ are assumed to have large absolute value $\left|S_{i}\right|=S \gg 1$. The characteristic $s$ - $d$ exchange energy is given by $\Delta=J S$. For conductivity and nonequilibrium spin density computations we assume the single-domain antiferromagnetic order, which is characterized by the unit Néel vector $\ell=\left(S^{A}-S^{B}\right) / 2 S$.

Equal exchange couplings on both sublattices in Eq. (5) and Rashba spin-orbit interaction of Eq. (3) ensure sublattice symmetry that greatly simplifies the results. In what follows we refer to the model as the symmetric model. At the end of the paper we also consider a more complex version of the model, where the sublattice symmetry is broken by a strong asymmetry of $s-d$ couplings on $A$ and $B$ sublattices.

The model of Eq. (1) is motivated, in part, by the studies of CuMnAs [21]. A similar model was also used to describe silicene where circularly polarized light induces a staggered $s-d$ interaction [54]. The spin-orbit interaction of Eq. (3) breaks $\hat{z} \rightarrow-\hat{z}$ inversion symmetry. Similarly to CuMnAs we also assume that the Fermi energy of conduction electrons stay in a vicinity of the antiferromagnetic gap (or Dirac point). That means that the Fermi surfaces are located near two different pockets of the Brillouin zone-the so-called $K$ and $K^{\prime}$ valleys as shown in Fig. 1(b) (see also the caption in Fig. 2).

Due to the rather high symmetry of the model of Eqs. (1)-(5), the Fermi surfaces remain entirely isotropic with respect to azimuthal (in-plane) direction of the Néel vector $\ell$ as illustrated in Fig. 1(b). Still, the band structure of the symmetric model depends strongly on the polar angle $\theta$ of the Néel vector, $\ell_{z}=\cos \theta$, as shown in Fig. 2 .

From the microscopic point of view all spin-orbit torques (as well as spin-transfer torques, spin-orbit induced Gilbert damping and effective renormalization of angular momenta $S_{i}$ ) can be directly related to nonequilibrium contributions to the local spin density $s_{i}$ that are proportional to electric current (or, in the case of Gilbert damping, to the time derivatives of 


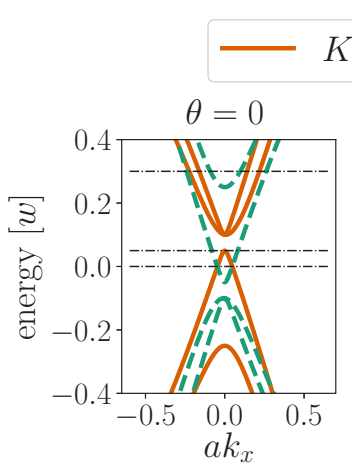

$$
K \text {-valley } \quad---\quad K^{\prime} \text {-valley }
$$
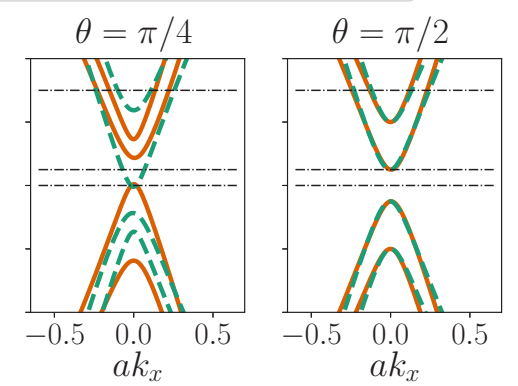

FIG. 2. The band structure cross section at $k_{y}=0$ for the model of Eq. (1) versus $k_{x}$ counted with respect to the high-symmetry points $\boldsymbol{K}$ and $\boldsymbol{K}^{\prime}$ for different orientations of the Néel vector, $\ell_{z}=\cos \theta$. Horizontal lines correspond to the Fermi energies $E=0.0 w, 0.05 w$, and $0.3 w$ for which we perform the numerical analysis. For zero energy (band center), the system is insulating for $\theta>\pi / 4$; for $\theta<$ $\pi / 4$ the Fermi energy crosses the conduction band in $K$ valley and the valence band in $K^{\prime}$ valley. For $E=0.05 w$ all extended states belong to the conduction band in $K$ valley that gives rise to $100 \%$ valley polarization. For $E=0.3 w$ both valleys contain two Fermi surfaces. The plots correspond to the parameters $\lambda=0.05 \mathrm{w}$ and $\Delta=0.1 w$.

the classical field $\boldsymbol{S}_{i}$ ) [55,56]. The microscopic analysis of the torques is, therefore, similar to the microscopic analysis of the conductivity and must involve the mechanisms of momentum relaxation of conduction electrons that, in our system, is also directly related to the angular-momentum relaxation of localized spins $\boldsymbol{S}_{i}$. Here we model such a momentum relaxation by adding nonmagnetic on-site disorder potential to the model of Eq. (1). Namely, we consider an ensemble of tight-binding models,

$$
H=H_{0}+\sum_{i} \sum_{\sigma \sigma^{\prime}} V_{i} c_{i \sigma}^{\dagger} c_{i \sigma^{\prime}},
$$

where $V_{i}$ are random on-site potentials on randomly selected sites. For numerical simulations we take $V_{i}= \pm V_{d}$ (with a random sign) on random lattice sites in the scattering region (the sample) as illustrated in Fig. 3. In various simulations we chose $V_{d}=0.2 \mathrm{w}$ and $V_{d}=0.5 \mathrm{w}$ and place impurities on 30, 40 , and $50 \%$ of the lattice sites in the sample. Each point is obtained by averaging over 30-80 disorder realizations.

Even though the spin-orbit torques are completely defined by the tight-binding model of Eq. (6), they are insufficient to describe magnetization dynamics of an antiferromagnet. The latter also depends on the type of the Heisenberg model used for $S_{i}$ as well as on the Gilbert damping terms. In the presence of magnetic textures one should also take into account inplane spin-transfer torques (which are defined by the response of spin density $\boldsymbol{s}_{i}$ to both electric current and spacial gradients of $\boldsymbol{S}_{i}$ ). Intimate relations between all these seemingly different (and often highly anisotropic) quantities have recently been established for a 2D Rashba ferromagnet in the metal regime [56].

\section{SCATTERING APPROACH}

In this paper we present a numerical analysis of spinorbit torques using the scattering framework. The framework

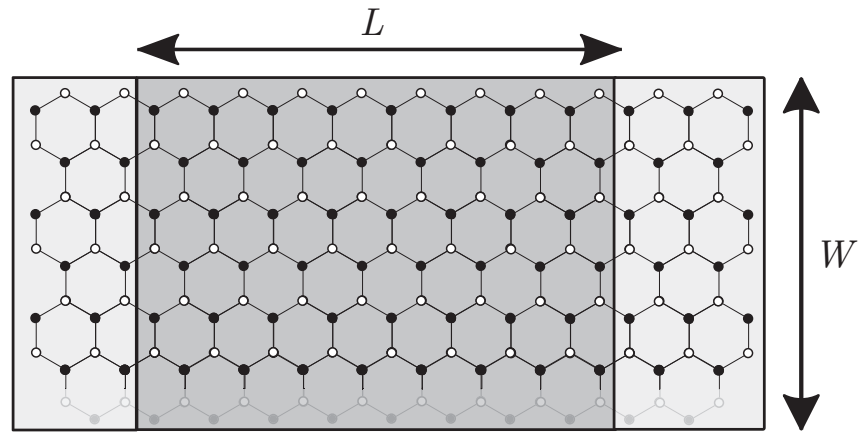

FIG. 3. Two-terminal geometry that is used for computation of spin-orbit torques. The largest sample corresponds to a wide zigzag nanoribbon with $L \sim W \sim 750 a$. We assume periodic boundary conditions in $y$ direction for both the sample and the leads. The sample is described by the model of Eq. (6) with on-site disorder potential $V_{i}= \pm V_{d}$. Left and right leads ( $x<0$ and $x>L$, respectively) are described by a clean Hamiltonian of Eq. (1). The nonequilibrium spin density per current flux (averaged over the entire sample) is defined by the linear response formula of Eq. (15).

appeals to the two terminal geometry depicted schematically in Fig. 3. Left and right leads (reservoirs) are modeled by semi-infinite systems of the width $W$ described by ballistic tight-binding model of Eq. (1) with periodic boundary conditions in $y$ direction. For both leads one constructs left- and right-going scattering states, $\Psi_{\alpha, E}^{L, \gtrless}\left(\boldsymbol{r}_{i}\right)$ and $\Psi_{\alpha, E}^{R, \gtrless}\left(\boldsymbol{r}_{i}\right)$, that are the eigenstates of the model of Eq. (1) that are normalized to the unit probability current flux through the lead cross section.

The scattering states are labeled by (i) the eigenenergy $E$; (ii) the lead index: $L$ for the left lead and $R$ for the right one; (iii) the flux direction: $>$ for the probability current in $x$ direction and $<$ for the probability current in the opposite $(-x)$ direction; and (iv) by a composite index $\alpha=(n, \sigma, v)$ that incorporates the channel index $n$ (numerating states with different projections of the wave vector on the transversal direction $y$ ), the spin projection $\sigma$ and the band index $v$ numerating Fermi surfaces. Note that the dimension of the scattering state wave function is $1 / \sqrt{W v_{\alpha}}$, where $v_{\alpha}$ is the $x$ component of the velocity in the channel $\alpha$.

With the help of the scattering states one can readily formulate a scattering problem at a given energy $E$ that is solved by the wave function matching at the lead-sample interface for each disorder realization. For example, a scattering problem that corresponds to populating an incoming channel $\Psi_{\alpha, E}^{L,>}$ results in the eigenstate that has the following form in the leads

$$
\Psi_{\alpha, E}^{L}(\boldsymbol{r})=\left\{\begin{array}{ll}
\Psi_{\alpha, E}^{L,>}(\boldsymbol{r})+\sum_{\beta} r_{\beta \alpha} \Psi_{\beta, E}^{L,<}(\boldsymbol{r}), & x<0, \\
\sum_{\beta} t_{\beta \alpha} \Psi_{\beta, E}^{R,>}(\boldsymbol{r}), & x>L
\end{array},\right.
$$

where $t_{\beta \alpha}$ and $r_{\beta \alpha}$ denote the so-called transmission and reflection amplitudes, correspondingly. Similarly, the scattering problem that corresponds to populating a left-going state in the right lead, $\Psi_{\alpha, E}^{R,<}$, corresponds to the eigenstate

$$
\Psi_{\alpha, E}^{R}(\boldsymbol{r})=\left\{\begin{array}{ll}
\sum_{\beta} t_{\beta \alpha}^{\prime} \Psi_{\beta, E}^{L,<}(\boldsymbol{r}), & x<0, \\
\Psi_{\alpha, E}^{R,<}(\boldsymbol{r})+\sum_{\beta} r_{\beta \alpha}^{\prime} \Psi_{\beta, E}^{R,>}(\boldsymbol{r}), & x>L
\end{array} .\right.
$$


The reflection and transmission amplitudes are organized into the scattering matrix

$$
S=\left(\begin{array}{cc}
\hat{r} & \hat{t}^{\prime} \\
\hat{t} & \hat{r}^{\prime}
\end{array}\right)
$$

that yields the unitarity constraint $S^{\dagger} S=1$. The constraint is ensured by the normalization of the scattering states $\Psi_{\alpha, E}^{\gtrless}(\boldsymbol{r})$ to the unit probability current flux that is conserved for each energy $E$ (in the absence of nonelastic processes).

The results of Eqs. (7) and (8) are routinely used to express, e.g., the time-averaged electric current flowing through the sample as

$$
I=e \int \frac{d E}{2 \pi \hbar}\left(f_{R}(E)-f_{L}(E)\right) \sum_{\alpha \beta}\left|t_{\alpha \beta}(E)\right|^{2},
$$

where $f_{L}$ and $f_{R}$ stand for electron distribution functions in the left and right leads, respectively, and $e=-|e|$ is the electron charge. The expression for electric current leads to the celebrated [57] Landauer-Büttiker formula for the conductance

$$
G=I / V_{\text {bias }}=\frac{e^{2}}{h} \sum_{\alpha \beta}\left|t_{\alpha \beta}(E)\right|^{2},
$$

where $V_{\text {bias }}$ is the voltage bias between the left and right leads (that sets a difference between the chemical potentials in the leads: $\left.\delta \mu=\mu_{L}-\mu_{R}=e V_{\text {bias }}\right)$ and $E$ is the average chemical potential in the sample (which we simply call the Fermi energy).

The dependence of the average conductance on the sample length (for $L \simeq W$ ) is then fitted by the following formula

$$
\langle G\rangle_{\mathrm{dis}}=\frac{2 e^{2}}{h} \frac{W}{L+\ell_{0}} \sigma,
$$

where the angular brackets stand for averaging over disorder realizations, while the constant $\sigma$ is regarded as 2D dimensionless conductivity (which is independent of both $L$ and $W$ ). The length scale $\ell_{0}$ in Eq. (12) is of the order of the transport mean free path in the system.

The straightforward numerical analysis using kwant package [51] provides us with the two terminal conductivity, $L\langle G\rangle / W$, that is plotted in Fig. 4 for the metal regime $(E=$ $0.3 \mathrm{w}$, left panel) and for the half-metal regime $(E=0.05 \mathrm{w}$, right panel). The metal regime refers here to the Fermi energies that correspond to two Fermi surfaces per valley, while the half-metal regime corresponds to a single Fermi surface. Similar plots are generated for various polar angles $\theta$ of the Neel vector. Both the 2D conductivity $\sigma$ and the mean free path $\ell_{0}$ are then extracted from the fitting formula of Eq. (12) and plotted in Fig. 5 as the function of $\ell_{z}=\cos \theta$. The estimate of $\ell_{0}$ is necessary to ensure that our sample size is at least of the order of the mean free path to avoid nonuniversal finite-size effects in our data.

One can see from Fig. 5 that both the mean free path $\ell_{0}$ and the conductivity $\sigma$ are nearly constant in the metal regime. Their dependence on the Néel vector orientation is weak, which is consistent with the fact that such a dependence must disappear in the limit $E \gg \Delta$. In the half-metal regime, however, the conductivity is strongly dependent on both disorder concentration and the Néel vector angle $\theta$. We will see

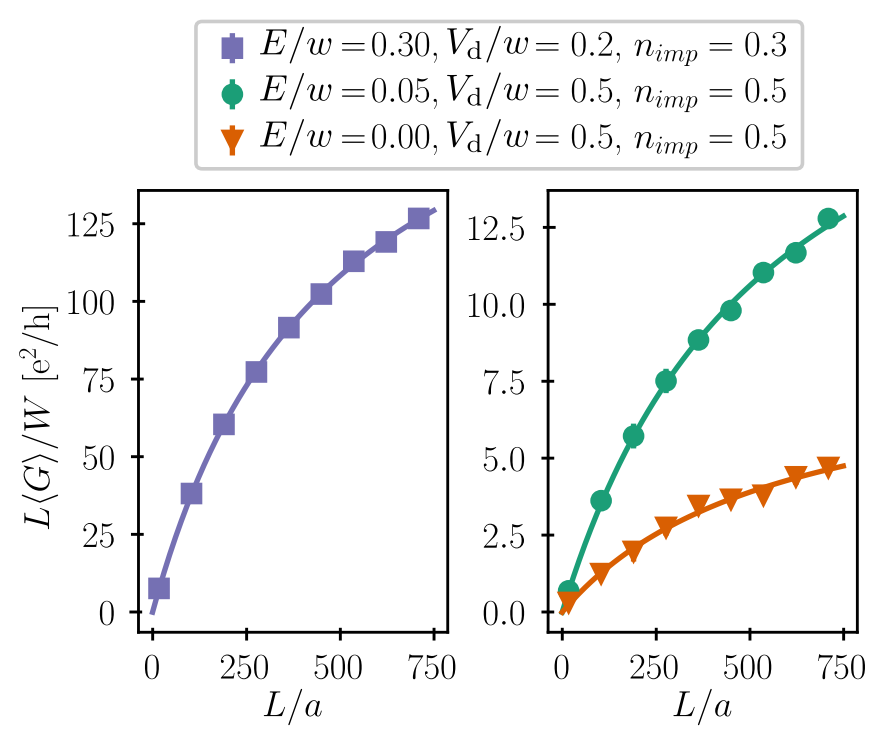

FIG. 4. Averaged two-terminal conductivity $L\langle G\rangle / W$ for the symmetric coupling model computed from Eq. (11) as the function of the sample length $L$ for several values of the Fermi energy $E$, impurity strength $V_{d}$ and impurity concentration $n_{\text {imp. }}$. Each point corresponds to averaging over 30 disorder realizations. The curves correspond to $\Delta=0.1 w, \lambda=0.05 w$, and $\theta=\pi / 4$ and are fitted using Eq. (12). Similar behavior is observed for various angles $\theta$.

below, that despite rather strong angular dependence of the conductivity, one of the fieldlike spin-orbit torques remains $\theta$ independent in the half-metal regime.

For $E=0$, the system exhibits metal-insulator transition as the function of the Néel vector orientation (see the second panel of Fig. 4). This choice of the Fermi energy leads, however, to vanishing spin-orbit torques as discussed below.

\section{SPIN-ORBIT TORQUES}

The package kwant [51] not only provides the elements of the scattering matrix $S(E)$ in Eq. (9) but also gives access to the solutions $\Psi_{\alpha, E}^{L, R}(\boldsymbol{r})$ inside the sample. Such solutions can therefore be used to obtain a local expectation value of the electron spin (we refer here to the dimensionless spin defined by the operator $\sigma / 2$ ) as

$$
\boldsymbol{s}(\boldsymbol{r})=\frac{1}{2} \int \frac{d E}{2 \pi \hbar} \sum_{A} f_{A}(E) \sum_{\alpha} \Psi_{\alpha, E}^{A \dagger} \boldsymbol{\sigma}_{\sigma \sigma^{\prime}} \Psi_{\alpha, E}^{A},
$$

where $\alpha=(n, \sigma, v)$ and the index $A=L, R$ numerates the leads.

In order to extract spin-orbit torques we have to decompose the local electron spin to equilibrium and nonequilibrium contributions as

$$
\boldsymbol{s}(\boldsymbol{r})=\boldsymbol{s}_{0}(\boldsymbol{r})+\delta \boldsymbol{s}(\boldsymbol{r})
$$

Equilibrium spin density, $\boldsymbol{s}_{0}$, corresponds to the limit of zero bias (or thermal bias) $f_{L}=f_{R}=f(E)$, where $f(E)$ is the Fermi-Dirac distribution function. The quantity $\boldsymbol{s}_{0}$ describes equilibrium conduction electron contributions to the parameters of the Heisenberg model for localized momenta $\boldsymbol{S}_{i}$, which we do not discuss here. 

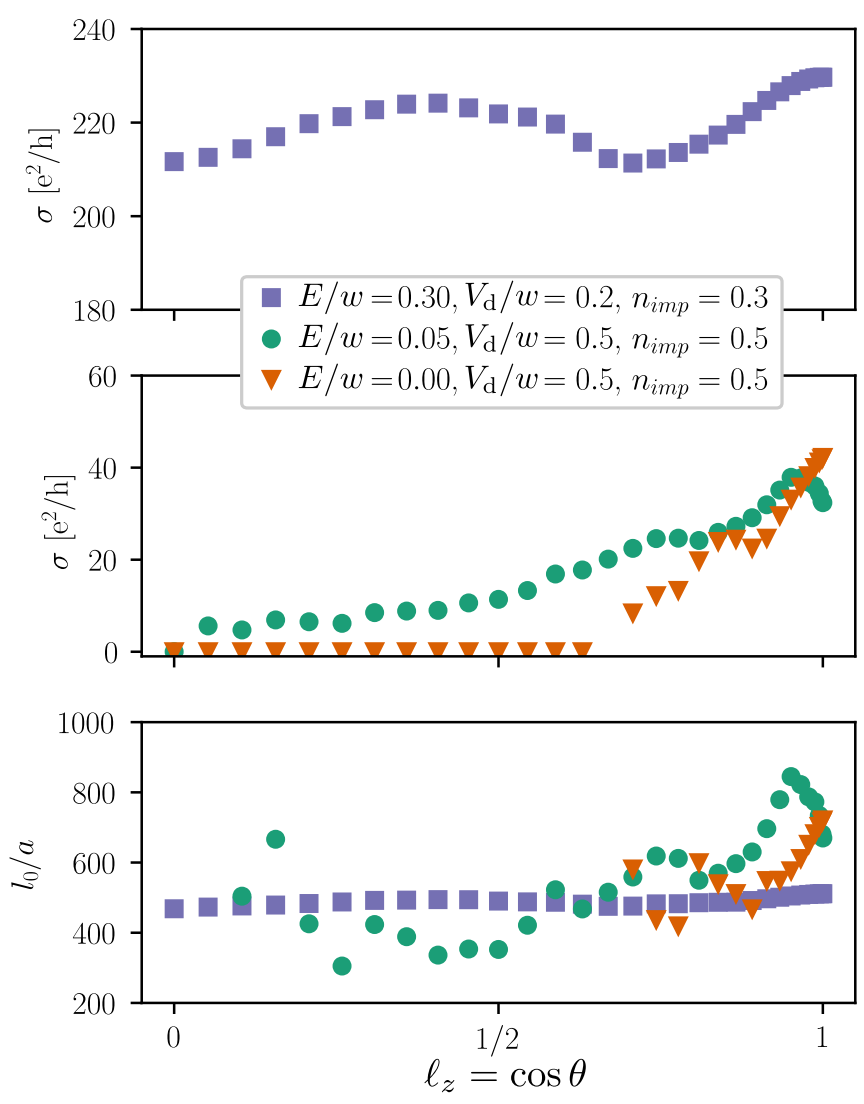

FIG. 5. The conductivity $\sigma$ and the mean free path $\ell_{0}$ in the symmetric coupling model of Eq. (6) as the function of the Néel vector orientation $\ell_{z}=\cos \theta$. Both quantities are extracted from our numerical data with the help of Eq. (12). Large variations of the mean free path in the half-metal regime are associated with the Fermi energy touching the band edge at certain polar angles of the Néel vector.

Nonequilibrium contribution, $\delta \boldsymbol{s}$, describes the effects induced, e.g., by a bias voltage, $V_{\text {bias }}$, such as the spin-orbit torques on magnetization. In the linear response we obtain, in the spirit of Eq. (11), the formula

$\frac{\delta \boldsymbol{s}(\boldsymbol{r})}{\delta \mu}=\frac{1}{4 h} \sum_{\alpha}\left[\Psi_{\alpha, E}^{R \dagger}(\boldsymbol{r}) \boldsymbol{\sigma}_{\sigma \sigma^{\prime}} \Psi_{\alpha, E}^{R}(\boldsymbol{r})-\Psi_{\alpha, E}^{L \dagger}(\boldsymbol{r}) \boldsymbol{\sigma}_{\sigma \sigma^{\prime}} \Psi_{\alpha, E}^{L}(\boldsymbol{r})\right]$,

where $\delta \mu=e V_{\text {bias }}$ and the scattering states are taken at the Fermi energy. Similarly to Eq. (11), we assume here that the quantities $\Psi_{\alpha, E}^{A \dagger} \sigma \Psi_{\alpha, E}^{A}$ have negligible energy dependence within the interval $\delta \mu$ around the Fermi energy.

In numerical simulations we systematically employ Eq. (15) to extract the spin density on $A$ and $B$ sublattices by varying the direction of the Néel vector. The results are fitted by the expansion of $\delta \boldsymbol{s}$ in angular harmonics that are compatible with the symmetry of the model. Before we proceed with the discussion of our results, let us pause to clarify the relation between the nonequilibrium spin-density and microscopic spin-orbit torques on magnetization that enter effective Landau-Lifshits-Gilbert (LLG) equation on magnetization dynamics.

\section{MICROSCOPIC LLG EQUATION}

The problem of magnetization dynamics can be formulated with the help of a Heisenberg model for the localized momenta $\boldsymbol{S}_{i}$ that is coupled to a tight-binding model for conduction electrons by means of the local exchange interaction of Eq. (5). Depending on the type of the Heisenberg model we may obtain different equations of motion but the torques on magnetization (originating from electric current or field) and also Gilbert damping terms can be understood from the tight-binding Hamiltonian of Eq. (6) alone [56].

For the sake of illustration let us outline the derivation of magnetization dynamics for a Heisenberg model of an "isotropic" antiferromagnet. The local interaction of Eq. (5) dictates the equation of motion of localized momenta of the form

$$
\dot{\boldsymbol{S}}_{i}=\boldsymbol{H}_{i} \times \boldsymbol{S}_{i}+\frac{J}{\hbar} \boldsymbol{S}_{i} \times \sum_{\sigma \sigma^{\prime}}\left\langle c_{i \sigma}^{\dagger} \boldsymbol{\sigma}_{\sigma \sigma^{\prime}} c_{i \sigma^{\prime}}\right\rangle,
$$

where dot stands for the time derivative of $\boldsymbol{S}_{i}$ and $\boldsymbol{H}_{i}=$ $-\delta F / \delta \hbar \boldsymbol{S}_{i}$ is an effective field (in frequency units) on a site $i$ that is defined by the free energy $F$ of the classical Heisenberg model for localized spins. The angular brackets denote the thermodynamic expectation value of conduction electron spin operator on the same site $i$.

For a collinear antiferromagnet we shall distinguish two sublattices $A$ and $B$ that are characterized by opposite directions of the localized momenta $S_{i}^{A}=-S_{i}^{B}$ in the antiferromagnetic ground state. It is assumed that these directions remain to be almost opposite even for out-of-equilibrium conditions. It is also natural to assume that, as far as the magnet is far from a phase transition, the classical fields $\boldsymbol{S}_{i}^{A}$ and $S_{i}^{B}$ remain smooth on atomic scales. Below we consider a single domain antiferromagnet, which is characterized by two time-dependent unit vectors $\boldsymbol{n}^{A, B}=\boldsymbol{S}^{A, B} / S$.

It is also convenient to define smooth conduction electron spin densities on each of the two sublattices

$$
\boldsymbol{s}^{A, B}(\boldsymbol{r})=\frac{1}{2} \sum_{i \sigma \sigma^{\prime}}\left\langle c_{i \sigma}^{\dagger} \boldsymbol{\sigma}_{\sigma \sigma^{\prime}} c_{i \sigma^{\prime}}\right\rangle \frac{2}{\mathcal{A}},
$$

where $\mathcal{A}$ is the area of the unit cell (which naturally includes one A and one B site). Thus, the equation of motion (16) can be written in continuous approximation as

$$
\begin{aligned}
\dot{\boldsymbol{n}}^{A} & =\boldsymbol{H}^{A} \times \boldsymbol{n}^{A}+(J \mathcal{A} / \hbar) \boldsymbol{n}^{A} \times \boldsymbol{s}^{A}, \\
\dot{\boldsymbol{n}}^{B} & =\boldsymbol{H}^{B} \times \boldsymbol{n}^{B}+(J \mathcal{A} / \hbar) \boldsymbol{n}^{B} \times \boldsymbol{s}^{B},
\end{aligned}
$$

where $\left|\boldsymbol{n}^{A, B}\right|=1$ and the notations $\boldsymbol{H}^{A, B}$ refer to the effective fields on the sublattices $A$ and $B$.

Antiferromagnet dynamics is usually formulated as the coupled dynamics of the Néel and magnetization vectors,

$$
\ell=\left(n^{A}-n^{B}\right) / 2, \quad m=\left(n^{A}+n^{B}\right) / 2,
$$

that remain mutually perpendicular $\boldsymbol{\ell} \cdot \boldsymbol{m}=0$ and yield the constraint $\ell^{2}+m^{2}=1$. Naturally, the amplitude of the magnetization vector remain to be small, $m \ll \ell \approx 1$.

For an "isotropic" antiferromagnet, one finds the effective field [7] $\boldsymbol{H}^{A}+\boldsymbol{H}^{B}=J_{\mathrm{ex}} \boldsymbol{m} / \hbar+2 \boldsymbol{H}$, where $\boldsymbol{H}$ is an external magnetic field in frequency units and $J_{\text {ex }}$ is a direct antiferromagnetic exchange energy that is one of the largest 
energies in the problem. In turn, the combination $\boldsymbol{H}^{A}-\boldsymbol{H}^{B}$ is proportional to magnetic anisotropy and we choose not to take into account as mentioned above.

Equations (18) can, therefore, be rewritten as

$$
\begin{aligned}
& \dot{\boldsymbol{\ell}}=-\frac{J_{\mathrm{ex}}}{2 \hbar} \boldsymbol{\ell} \times \boldsymbol{m}+\boldsymbol{H} \times \boldsymbol{\ell}+\frac{J \mathcal{A}}{\hbar}\left(\boldsymbol{\ell} \times \boldsymbol{s}_{+}+\boldsymbol{m} \times \boldsymbol{s}_{-}\right), \\
& \dot{\boldsymbol{m}}=\boldsymbol{H} \times \boldsymbol{m}+\frac{J \mathcal{A}}{\hbar}\left(\boldsymbol{m} \times \boldsymbol{s}_{+}+\boldsymbol{\ell} \times \boldsymbol{s}_{-}\right),
\end{aligned}
$$

where $\boldsymbol{s}_{ \pm}=\left(\boldsymbol{s}_{A} \pm \boldsymbol{s}_{B}\right) / 2$. The right-hand sides of Eqs. (20) contain the quantities that can be called generalized torques. Here we are only interested in specific contributions to generalized torques that are induced by the chemical potential difference $\delta \mu=e V_{\text {bias }}$ between left and right leads. Such contributions define four spin-orbit torques

$$
\boldsymbol{T}_{ \pm}^{\ell}=(J \mathcal{A} / \hbar) \boldsymbol{\ell} \times \delta \boldsymbol{s}_{ \pm}, \quad \boldsymbol{T}_{ \pm}^{m}=(J \mathcal{A} / \hbar) \boldsymbol{m} \times \delta \boldsymbol{s}_{ \pm},
$$

where $\delta \boldsymbol{s}_{ \pm}$refers to the nonequilibrium spin density contribution that is proportional to $\delta \mu$.

Note that, generally, the average spin $s_{i}$ has a nonlocal functional dependence on the time-dependent classical field $\boldsymbol{S}_{j}$ at preceding moments of time and at different lattice sites $j \neq i$. The degree of nonlocality is defined by relaxation processes. The quicker the relaxation the more local the functional dependence. In particular, nondissipative contributions to spin-orbit torques (the so-called fieldlike contributions) are local in time on the timescales of the order of $s-d$ exchange $\tau_{\mathrm{sd}}=\hbar / \Delta$, where $\Delta=J S$. In contrast, the dissipative contributions (such as antidamping torques) are defined by transport scattering time $\tau_{\text {tr }}$. The latter timescale may be both larger and smaller than $\tau_{\mathrm{sd}}$ giving rise to different regimes of magnetization dynamics. For the sake of numerical analysis we consider, however, a situation when these two timescales are of the same order.

\section{SYMMETRY CONSIDERATION}

Symmetry properties of spin-orbit torques and conductivity tensor can be understood from a low-energy approximation by projecting the model (1) on the states in a vicinity of $K$ points,

$$
\boldsymbol{K}=\frac{4 \pi}{3 \sqrt{3} a}\left(\begin{array}{l}
1 \\
0
\end{array}\right), \quad \text { and } \quad \boldsymbol{K}^{\prime}=-\boldsymbol{K},
$$

as is usually done for graphene. In the valley symmetric approximation we obtain the effective model

$$
H_{0}^{\text {eff }}=v \boldsymbol{p} \cdot \boldsymbol{\Sigma}+\alpha_{R}[\boldsymbol{\sigma} \times \boldsymbol{\Sigma}]_{\hat{z}}-\Delta \boldsymbol{\ell} \cdot \boldsymbol{\sigma} \Sigma_{z} \Lambda_{z},
$$

where $\boldsymbol{\Sigma}, \boldsymbol{\Lambda}$, and $\sigma$ are the vectors of Pauli matrices in sublattice, valley, and spin space, respectively, and

$$
v=\frac{3}{2} w a / \hbar, \quad \alpha_{R}=\frac{3}{2} \lambda, \quad \Delta=J S .
$$

Even though the model of Eq. (1) is characterized by the point group $C_{3 v}$, the effective low-energy model of Eq. (23) has a higher symmetry of the group $C_{\infty v}$. This is reflected by the fact that the Fermi surfaces (for energies we are interested in) remain entirely isotropic irrespective of the Néel vector orientation as shown in Fig. 1. Moreover, the exact sublattice symmetry of the model, $\Lambda_{x} H_{0}^{\text {eff }}[-\ell] \Lambda_{x}=H_{0}^{\text {eff }}[\ell]$, ensures vanishing nonequilibrium staggered spin polarization $\delta \boldsymbol{s}_{-}=0$ irrespective of both the voltage bias and the Néel vector orientation. This property is indeed confirmed by the numerical analysis.

The spin-orbit coupling term in Eq. (23) is proportional to the scalar product $\sigma \cdot[\hat{z} \times \boldsymbol{v}]$, where $\boldsymbol{v}=v \boldsymbol{\Sigma}$ is the velocity operator and $\hat{z}$ is the unit vector in $z$ direction. In the presence of electric current $I$, which, in our model, is always directed along $x$ axis, the velocity operator averages to a nonzero value that is proportional to the current. One can see, therefore, that the second term in Eq. (23) can be interpreted as Zeeman coupling to an effective Rashba field $\hat{z} \times \boldsymbol{I}$. Such a field induces nonequilibrium polarization $\delta \boldsymbol{s}_{+}$in the same way as in ferromagnets.

The symmetry of the point group $C_{\infty v}$ (see also the symmetry analysis in Refs. [58,59]) suggests that the tensor relation between nonequilibrium polarization $\delta \boldsymbol{s}_{+}$and the Rashba field $\hat{z} \times \boldsymbol{I}$ can be presented in a vector form as

$$
\begin{aligned}
\delta \boldsymbol{s}_{+}= & A_{I}\left(\ell_{z}^{2}\right) \hat{z} \times \boldsymbol{I}+A_{I}^{\prime}\left(\ell_{z}^{2}\right) \boldsymbol{\ell}_{\|} \times\left[\boldsymbol{\ell}_{\|} \times[\hat{z} \times \boldsymbol{I}]\right] \\
& +B_{\perp}\left(\ell_{z}^{2}\right) \ell_{\perp} \times[\hat{z} \times \boldsymbol{I}]+B_{\|}\left(\ell_{z}^{2}\right) \boldsymbol{\ell}_{\|} \times[\hat{z} \times \boldsymbol{I}] \\
& +C\left(\ell_{z}^{2}\right) \boldsymbol{\ell}_{\|} \times\left[\boldsymbol{\ell}_{\perp} \times[\hat{z} \times \boldsymbol{I}]\right],
\end{aligned}
$$

where we decompose the Néel vector $\boldsymbol{\ell}=\boldsymbol{\ell}_{\|}+\boldsymbol{\ell}_{\perp}$ into in-plane $\ell_{\|}$and perpendicular-to-the-plane $\ell_{\perp}$ components. We parametrize $\ell=(\cos \phi \sin \theta, \sin \phi \sin \theta, \cos \theta)^{\top}$, where $\theta$ is a polar angle and $\phi$ is an azimuthal angle. Consequently, we have $\boldsymbol{\ell}_{\|}=(\cos \phi \sin \theta, \sin \phi \sin \theta, 0)^{\top}$ and $\boldsymbol{\ell}_{\perp}=$ $(0,0, \cos \theta)^{\top}$.

The decomposition of Eq. (25) is dictated by the symmetry analysis with respect to two transformations: The Néel vector inversion, $\ell \rightarrow-\ell$, and the Néel vector reflection, $\ell_{\perp} \rightarrow$ $-\ell_{\perp}$, with respect to the electron $2 \mathrm{D}$ plane.

Since the model (at the Fermi energies we choose) is isotropic with respect to in-plane rotations, these two symmetries are the only ones to characterize different contributions to nonequilibrium spin density.

The first two terms in Eq. (25) are even with respect to both the Néel vector inversion and the Néel vector reflection. The last term in Eq. (25) is even with respect to the Néel vector inversion but odd with respect to the Néel vector reflection. These terms correspond to torques of the fieldlike symmetry, i.e., to the torques that are invariant under the time reversal operation.

The second two terms in Eq. (25), which are proportional to the coefficients $B_{\|, \perp}$, are odd with respect to the Néel vector reflection. The first one of them is odd while the second one is even with respect to the Néel vector reflection. These terms represent antidamping spin-orbit torques that change sign under time reversal.

The coefficients in front of different vector forms in Eq. (25) must remain invariant with respect to both symmetry transformations, but they may still vary as the function of the symmetry invariant $\ell_{z}^{2}$. The coefficients may also depend on $\ell^{2}$ but such dependence is absent within linear response approximation. Indeed, one shall take $\ell^{2}=1(m=0)$ in all coefficients in front of electric current since electric current is regarded as the only possible source of a deviation from the exact antiferromagnetic order. 
The exact sublattice symmetry of the symmetric coupling model of Eqs. (1)-(6) does not only ensure the vanishing staggered polarization $\delta \boldsymbol{s}_{-}=0$ but also leads to identically vanishing antidamping torques $B_{\|}=B_{\perp}=0$ in all possible regimes. This is indeed confirmed by our numerical analysis for the symmetric coupling model as we describe below.

\section{RESULTS FOR SYMMETRIC MODEL}

\section{A. General}

With the help of the kwant package [51] we compute from Eq. (15) the mean spin density response $\delta s / \delta \mu$ on both sublattices for various directions of the Néel vector $\ell$ and three different Fermi energies. These results, which are presented in Fig. 6, have complex dependence on the orientation of the Néel vector. The dependence is, however, much simplified for nonequilibrium spin density response divided by the sample conductance. In other words, we observe that the nonequilibrium spin density has a much simpler form when expressed via the charge current density $\boldsymbol{j}=\boldsymbol{I} / W$ rather than via the bias voltage.

In all regimes considered we find $\delta \boldsymbol{s}_{-}=0$ and confirm the decomposition of Eq. (25) with $B_{\|}=B_{\perp}=0$. Moreover, we also find that the coefficient $A_{I}\left(\ell_{z}^{2}\right)=A_{I}$ is constant, i.e., independent of the angle $\theta$ in all regimes we consider. In addition, we find that the nonequilibrium spin density vanishes identically, $\delta \boldsymbol{s}_{+}=\delta \boldsymbol{s}_{-}=0$ for $E=0$. This point corresponds to the exact electron-hole symmetry of the model, i.e., for

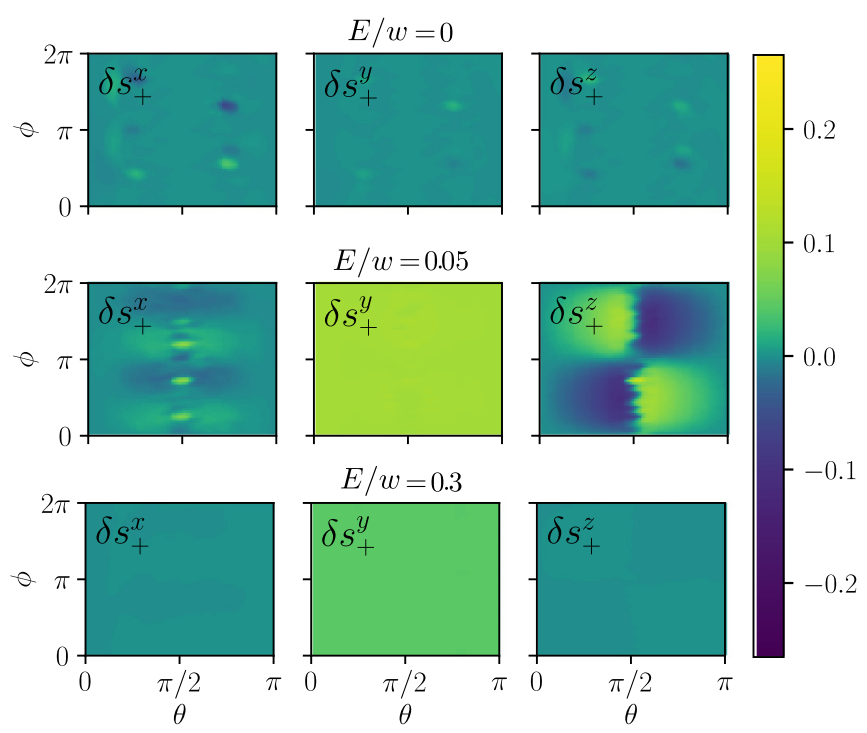

FIG. 6. Nonequilibrium spin density $\delta s_{+}$for the symmetric coupling model of Eqs. (1)-(6) with $V_{d}=0.5 \mathrm{w}$ as the function of the Néel-vector orientation for different values of the Fermi energy. Each data point foe each direction of the Néel vector corresponds to averaging over 30 impurity configurations. Spin densities are normalized to the scale $\Delta \lambda j / e v w^{2}$, where $j=I / W$ is the charge current density through the sample. Fluctuations at $\theta=\pi / 4$ and $\theta=3 \pi / 4$ in the top panel and at $\theta=\pi / 2$ in the middle panel are due to diverging mean free path for Fermi energy touching the bottom of the conduction band. The staggered spin density $\delta \boldsymbol{s}_{-}$fluctuates around zero (not-shown). For $E / w=0$, the spin-density $\delta \boldsymbol{s}_{+}$also fluctuates around zero as shown in the upper panel.
$E=0$, the number of quasiparticles in the conduction and valence bands are exactly the same.

Despite these general findings we still observe that the results for nonequilibrium spin density are qualitatively different in the metal and half-metal regimes.

\section{B. Metal regime}

The metal regime is characterized by two Fermi surfaces for each of the two valleys. This regime is represented in our simulations by the choice $E=0.3 \mathrm{w}$. In this case we also find $A_{I}^{\prime}=C=0$ within our numerical accuracy. Thus, the metal regime in the symmetric model is represented by the only contribution to the nonequilibrium spin-density,

$$
\delta \boldsymbol{s}_{+}=\frac{\Delta \lambda}{2 \pi e v w^{2}} a_{I} \hat{z} \times \boldsymbol{j},
$$

with a constant dimensionless coefficient $a_{I}$. This coefficient retains almost the same value for three different impurity concentrations as shown in the left panel of Fig. 7. Thus, the nonequilibrium spin density in the metal regime is simply proportional to the Rashba field. The nonequilibrium spin density of Eq. (26) is readily recognized as the inverse spingalvanic effect of Edelstein [60] that is widely known in ferromagnet materials with Rashba coupling [61,62].

Consequently, the spin orbit torques in the metal regime of the symmetric model are given solely by the isotropic Edelstein effect as

$$
\boldsymbol{T}_{+}^{\ell}=a_{I} \eta \boldsymbol{\ell} \times[\hat{z} \times \boldsymbol{j}], \quad \boldsymbol{T}_{+}^{m}=a_{I} \eta \boldsymbol{m} \times[\hat{z} \times \boldsymbol{j}],
$$

where $\eta=J \mathcal{A} \Delta \lambda / e v h w^{2}$.

The results of Eqs. (26) and (27) are remarkably similar to those found analytically in the metal regime of a Rashba ferromagnet model with white-noise disorder $[55,56]$. The latter is also characterized by identically vanishing antidamping spinorbit torques and by a completely isotropic fieldlike torque of the same symmetry. Even though, analytical results have been obtained for a ferromagnet with a weak white-noise disorder, the same drastic simplification of the spin-orbit torque takes place for a symmetric honeycomb antiferromagnet with rather strong pointlike disorder as demonstrated above. Such a simplification is, however, limited to the metal regime.

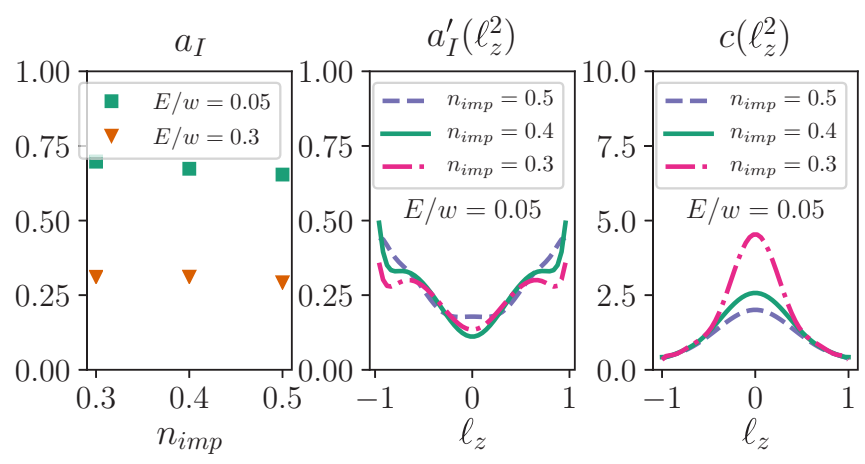

FIG. 7. The results of fitting simulation data for the symmetric coupling model with $L=2 W=750 a$ (partly shown in Fig. 6) with the ansatz of Eq. (28). The data are obtained for $V_{d}=0.5 \mathrm{w}$. The data have been collected for 200 different orientations of the Néel vector for each impurity concentration. 


\section{Half-metal regime}

The half-metal regime is represented by the Fermi energy $E=0.05 w$ that corresponds to the presence of a single Fermi surface in one of the valleys. The conductivity in this regime acquires strong dependence on $\theta$. In particular, for $\ell_{z}^{2}<1 / 2$, the system is poorly conducting as one can see already in the second panel of Fig. 5. Remarkably, we still find that the coefficient $A_{I}$ in Eq. (25) is entirely independent of $\theta$ and antidamping like torques are vanishing identically, $B_{\|, \perp}=0$. The coefficients $A_{I}^{\prime}$ and $C$ are, however, finite and acquire some dependence on $\ell_{z}^{2}$.

Thus, our numerical data in half-metal regime corresponds to the spin density

$$
\begin{aligned}
\delta \boldsymbol{s}_{+}= & \frac{\Delta \lambda}{2 \pi e v w^{2}}\left(a_{I} \hat{z} \times \boldsymbol{j}+a_{I}^{\prime}\left(\ell_{z}^{2}\right) \boldsymbol{\ell}_{\|} \times\left[\boldsymbol{\ell}_{\|} \times[\hat{z} \times j]\right]\right. \\
& \left.+c\left(\ell_{z}^{2}\right) \boldsymbol{\ell}_{\|} \times\left[\ell_{\perp} \times[\hat{z} \times j]\right]\right),
\end{aligned}
$$

where we also introduced the dimensionless functions $a_{I}^{\prime}\left(\ell_{z}^{2}\right)$ and $c\left(\ell_{z}^{2}\right)$ that are shown in the middle and in the right panel of Fig. 7 for different impurity concentrations.

The last two terms in Eq. (28) represent high-harmonics fieldlike torques that are finite only in the half-metal regime. On symmetry grounds one should generally expect the fieldlike torques to be largely insensitive to disorder. Even though, the disorder dependence of $a_{I}$ and $a_{I}^{\prime}$ coefficients is indeed negligible, the one of the function $c\left(\ell_{z}^{2}\right)$ is still rather strong for $\ell_{z}^{2}<1 / 2$. One should, however, remember that the coefficient $c$ is standing in front of the vector form that is vanishing for both $\ell_{z}=0$ and $\ell_{z}= \pm 1$, so the fit accuracy near these points is poor. Moreover, the case of almost in-plane Néel vector, $\ell_{z}^{2} \ll 1 / 2$, corresponds to the poorly conducting sample whose conductance is dominated by the variable range hopping processes due to strong disorder. Thus, we attribute this seemingly strong dependence of $c$ on disorder concentration to the mechanism of conduction. Still, the value of $c$ is found to be about 5 times larger than that of $a_{I}$ and about 10 times larger than that of $a_{I}^{\prime}$, which makes the high-harmonic $c$-torque relevant in the half-metal regime. We note that the nonequilibrium spin density, which is proportional to $c\left(\ell_{z}^{2}\right)$, is directed perpendicular to the electron plane.

Overall, the half-metal regime is characterized by the torques

$$
\begin{aligned}
\boldsymbol{T}_{+}^{\ell}= & \eta\left(a_{I} \boldsymbol{\ell} \times[\hat{z} \times \boldsymbol{j}]+a_{I}^{\prime}\left(\ell_{z}^{2}\right) \boldsymbol{\ell} \times\left[\boldsymbol{\ell}_{\|} \times\left[\boldsymbol{\ell}_{\|} \times[\hat{z} \times \boldsymbol{j}]\right]\right]\right. \\
& \left.+c\left(\ell_{z}^{2}\right) \boldsymbol{\ell} \times\left[\boldsymbol{\ell}_{\|} \times\left[\boldsymbol{\ell}_{\perp} \times[\hat{z} \times \boldsymbol{j}]\right]\right]\right), \\
\boldsymbol{T}_{+}^{m}= & \eta\left(a_{I} \boldsymbol{m} \times[\hat{z} \times \boldsymbol{j}]+a_{I}^{\prime}\left(\ell_{z}^{2}\right) \boldsymbol{m} \times\left[\boldsymbol{\ell}_{\|} \times\left[\boldsymbol{\ell}_{\|} \times[\hat{z} \times \boldsymbol{j}]\right]\right]\right. \\
& \left.+c\left(\ell_{z}^{2}\right) \boldsymbol{m} \times\left[\boldsymbol{\ell}_{\|} \times\left[\boldsymbol{\ell}_{\perp} \times[\hat{z} \times \boldsymbol{j}]\right]\right]\right),
\end{aligned}
$$

where $\eta=J \mathcal{A} \Delta \lambda / e v h w^{2}$ is the same as in Eq. (27).

Note that the function $a_{I}^{\prime}\left(\ell_{z}^{2}\right)$ in Eq. (29a) for $\boldsymbol{T}_{+}^{\ell}$ can be absorbed into the redefinition of the coefficients $a_{I}$ and $c$. Indeed, with the help of a straightforward vector algebra one can rewrite Eq. (29a) as

$$
\boldsymbol{T}_{+}^{\ell}=\eta\left(\tilde{a}_{I}\left(\ell_{z}^{2}\right) \boldsymbol{\ell} \times[\hat{z} \times \boldsymbol{j}]+\tilde{c}\left(\ell_{z}^{2}\right) \boldsymbol{\ell} \times\left[\boldsymbol{\ell}_{\|} \times\left[\boldsymbol{\ell}_{\perp} \times[\hat{z} \times \boldsymbol{j}]\right]\right]\right),
$$

where we introduced

$$
\tilde{a}_{I}=a_{I}-\left(1-\ell_{z}^{2}\right) a_{I}^{\prime}, \quad \tilde{c}=c-a_{I}^{\prime} .
$$

Thus, the modified coefficient $\tilde{a}_{I}$ acquires a weak dependence on $\ell_{z}^{2}$ due to a finite, though small, $a_{I}^{\prime}$ contribution in the halfmetal regime.

To conclude this section it is worth mentioning that halfmetal antiferromagnets are not entirely hypothetical. There have been several proposals in the past (see Refs. [63,64] to name a few) and there has been also a recent material $\mathrm{Mn}_{2} \mathrm{Ru}_{\mathrm{x}} \mathrm{Ga}$ that is recognized as a half-metal antiferromagnet $[65,66]$.

\section{ASYMMETRIC COUPLING MODEL}

Relative simplicity of the results of Eqs. (27) and (29) for the symmetric coupling model of Eqs. (1)-(6) prompted us to look at a model with ultimately asymmetric $s$ - $d$ exchange,

$$
H_{\mathrm{sd}}=-J \sum_{i \in A} \sum_{\sigma \sigma^{\prime}} \boldsymbol{\sigma}_{\sigma \sigma^{\prime}} \boldsymbol{S}_{i}^{A} \cdot c_{i \sigma}^{\dagger} c_{i \sigma^{\prime}}
$$

that is represented by $s-d$ coupling on $A$ sublattice only. Such a coupling obviously violates the sublattice symmetry and may, in principle, lead to the appearance of nonequilibrium staggered polarization and to antidamping like spin-orbit torques that are absent in the symmetric coupling model.

It is worth mentioning that the asymmetric model is unlikely to represent an antiferromagnet. Since conduction electron spins contribute to polarization only on a single sublattice, the model is much more likely to correspond to a ferrimagnet or a ferromagnet. Rare-earth-metal/transitionmetal ferrimagnets (such as FeCoGd) at a compensation point seem to be the most obvious examples [12]. The conduction electrons in these compounds couple mostly with localized $d$ orbitals rather than with $f$ orbitals and give rise to an asymmetric exchange coupling that can be mimicked by Eq. (32).

In order to compute nonequilibrium spin densities we repeat the analysis of the previous sections for the coupling of Eq. (32) that we refer to below as the asymmetric model. The band structure of the asymmetric model is illustrated in Fig. 8. Similarly to the symmetric model we distinguish metal and half-metal regimes that are represented now by $E=0.3 w$ and $E=0.1 \mathrm{w}$, correspondingly.

The metal regime is characterized by two Fermi surfaces per valley, while the half-metal regime is characterized by a single Fermi surface per valley. The Fermi surfaces are illustrated in Fig. 9 for $\theta=-\phi=\pi / 4$, where $\theta$ and $\phi$ are

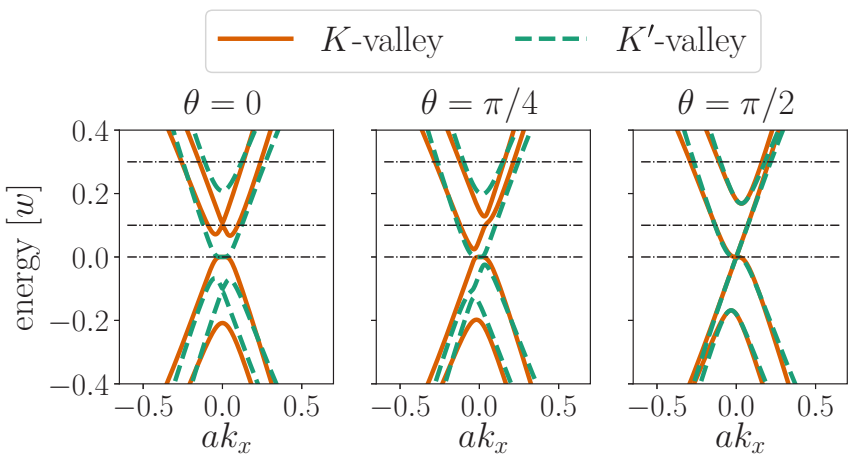

FIG. 8. Band structure for the asymmetric model. Dotted horizontal lines correspond to $E / t=0,0.1$, and 0.3 . 

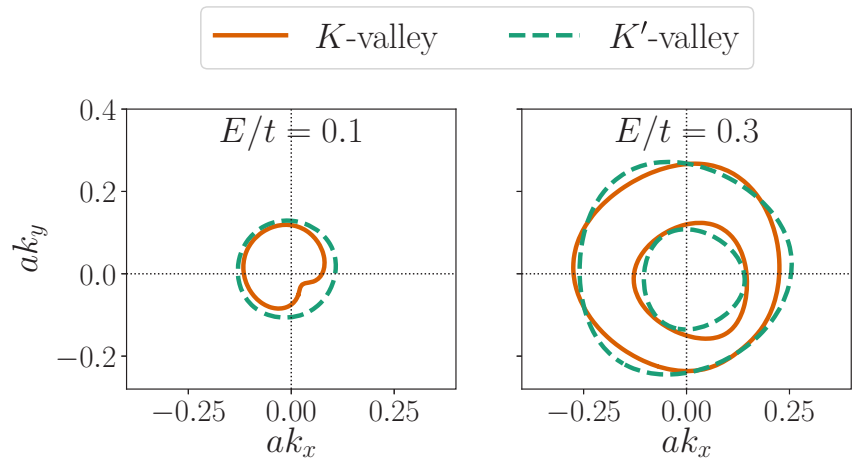

FIG. 9. The Fermi surfaces for the asymmetric model for the metal regime $E=0.3 w$ (right panel) and the half-metal regime $E=0.1 w$ (left panel). The Fermi surfaces are not round due to tridiagonal wrapping originating in $C_{3 v}$ point-group symmetry of the crystal and due to in-plane component $\boldsymbol{n}_{\|}^{A}$ that affects the spectrum. The plots correspond to the choice $\theta=-\phi=\pi / 4$. The momentum $\boldsymbol{k}$ is measured with respect to $\boldsymbol{K}$ point (solid lines) and $\boldsymbol{K}^{\prime}$ point (dashed lines).

now the polar and azimuthal angles of the unit vector $\boldsymbol{n}^{A}=$ $(\cos \phi \sin \theta, \sin \phi \sin \theta, \cos \theta)^{\top}$, correspondingly.

For numerical simulations we choose $\Delta=J S=0.1 w$, $\lambda=0.05 w$, and $V_{d}=0.5 w$ as for the symmetric model.

Similarly to Eq. (23) the low-energy sector of the asymmetric model is represented by the following effective Hamiltonian:

$$
H_{0}^{\text {as }}=v \boldsymbol{p} \cdot \boldsymbol{\Sigma}+\alpha_{R}[\boldsymbol{\sigma} \times \boldsymbol{\Sigma}]_{\hat{z}}-\frac{\Delta}{2} \boldsymbol{n}^{A} \cdot \boldsymbol{\sigma}\left(1+\Sigma_{z} \Lambda_{z}\right)
$$

that clearly lacks the sublattice symmetry. Interestingly, we observe from numerical simulations that the staggered nonequilibrium polarization $\delta \boldsymbol{s}_{-}$is still vanishing in the metal regime, while it becomes finite in the half-metal regime. It has to be noted, however, that the separation to staggered and nonstaggered polarization is largely irrelevant for the asymmetric model, since the latter is characterized by the only vector torque

$$
\boldsymbol{T}^{A}=\frac{J \mathcal{A}}{\hbar} \boldsymbol{n}^{A} \times \delta \boldsymbol{s}^{A}=\frac{J \mathcal{A}}{\hbar}(\boldsymbol{\ell}+\boldsymbol{m}) \times\left(\delta \boldsymbol{s}_{+}+\delta \boldsymbol{s}_{-}\right),
$$

that is defined exclusively by the spin density on the sublattice A.

Indeed, for an "isotropic" antiferromagnet we find, in complete analogy with Eqs. (20), the equations of motion

$$
\begin{aligned}
\dot{\boldsymbol{\ell}} & =-\left(J_{\mathrm{ex}} / 2 \hbar\right) \boldsymbol{\ell} \times \boldsymbol{m}+\boldsymbol{H} \times \boldsymbol{\ell}+\boldsymbol{T}^{A}, \\
\dot{\boldsymbol{m}} & =\boldsymbol{H} \times \boldsymbol{m}+\boldsymbol{T}^{A},
\end{aligned}
$$

which depend only on the spin-orbit torque $\boldsymbol{T}^{A}$.

We note that the equations of motion of Eq. (35) are essentially incomplete since we do not investigate Gilbertdamping terms and we do not take into account anisotropy of antiferromagnet (as well as Dzyaloshinskii-Moriya interaction terms). The missing terms are essential if one wants to understand the magnetization dynamics, while they cannot alter the spin-orbit torque terms that we compute.

As one can readily see from Fig. 9, the Fermi surfaces in the asymmetric model are not entirely round and reveal

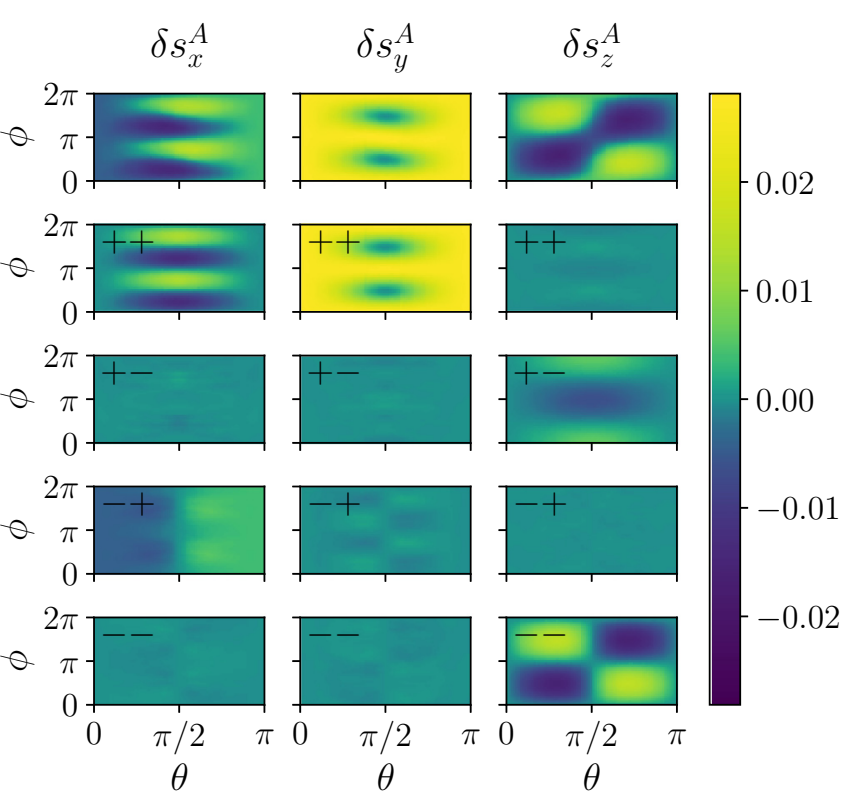

FIG. 10. Top panels show nonequilibrium spin density, $\delta \boldsymbol{s}^{A}$, for the asymmetric coupling model with subtracted background component along $\boldsymbol{n}^{A}$ in the units of $\Delta \lambda j / e v w^{2}$. The other panels represent the results of the spin-density decomposition of Eq. (36). The results presented correspond to the ansatz of Eq. (38). The data for density plots have been collected for 200 different orientations of the Néel vector for each impurity concentration. Each data point corresponds to averaging over at least 80 impurity configurations.

some tridiagonal wrapping even for energies that are close to zero, $E \ll w$. The Fermi surfaces are also shifted with respect to the points $\boldsymbol{K}$ and $\boldsymbol{K}^{\prime}$ and deformed depending on the azimuthal angle $\phi$ characterizing in-plane orientation of the vector $\boldsymbol{n}^{A}$. We find, however, that despite these effects the nonequilibrium spin-density $\delta \boldsymbol{s}^{A}$ can be very well decomposed using the symmetry analysis of the group $C_{\infty v}$ in analogy to Eq. (25) for the symmetric coupling model.

To do that we represent the vector $\boldsymbol{n}^{A}=\boldsymbol{n}_{\|}^{A}+\boldsymbol{n}_{\perp}^{A}$ as the sum of in-plane and perpendicular-to-the-plane components and decompose the numerical data with respect to the transformations: $\boldsymbol{n}_{\perp}^{A} \rightarrow-\boldsymbol{n}_{\perp}^{A}$ and $\boldsymbol{n}_{\|}^{A}=-\boldsymbol{n}_{\|}^{A}$. As a result, the nonequilibrium spin density (as the function of the vector components) is readily decomposed into the sum of four contributions,

$$
\delta \boldsymbol{s}^{A}\left[\boldsymbol{n}_{\perp}^{A}, \boldsymbol{n}_{\|}^{A}\right]=\delta \boldsymbol{s}_{++}+\delta \boldsymbol{s}_{+-}+\delta \boldsymbol{s}_{-+}+\delta \boldsymbol{s}_{--},
$$

which are expressed as

$$
\begin{aligned}
\delta \boldsymbol{s}_{\zeta \kappa}= & \frac{1}{4}\left(\delta \boldsymbol{s}^{A}\left[\boldsymbol{n}_{\perp}^{A}, \boldsymbol{n}_{\|}^{A}\right]+\zeta \delta \boldsymbol{s}^{A}\left[-\boldsymbol{n}_{\perp}^{A}, \boldsymbol{n}_{\|}^{A}\right]\right. \\
& \left.+\kappa \delta \boldsymbol{s}^{A}\left[\boldsymbol{n}_{\perp}^{A},-\boldsymbol{n}_{\|}^{A}\right]+\zeta \kappa \delta \boldsymbol{s}^{A}\left[-\boldsymbol{n}_{\perp}^{A},-\boldsymbol{n}_{\|}^{A}\right]\right),
\end{aligned}
$$

where $\zeta$ and $\kappa$ take on the values \pm 1 .

The bare results for nonequilibrium spin density $\delta \boldsymbol{s}^{A}$ (with a subtracted background component along $\boldsymbol{n}^{A}$ ) and the results for the contributions $\delta \boldsymbol{s}_{\zeta \kappa}$ are shown in Fig. 10 for the metal regime, $E=0.3 w$. Similar results are obtained for the halfmetal regime, $E=0.1 w$.

We find that our numerical results for the nonequilibrium spin density $\delta \boldsymbol{s}^{A}$ (with subtracted background along $\boldsymbol{n}^{A}$ that 
does not enter the torque $\boldsymbol{T}^{A}$ ) are perfectly decomposed as a sum of five different vector forms in both metal and half-metal regimes,

$$
\begin{aligned}
\delta \boldsymbol{s}^{A}= & A_{I}\left(n_{z}^{2}\right) \hat{z} \times \boldsymbol{I}+A_{I}^{\prime}\left(n_{z}^{2}\right) \boldsymbol{n}_{\|}^{A} \times\left[\boldsymbol{n}_{\|}^{A} \times[\hat{z} \times \boldsymbol{I}]\right] \\
& +B_{\perp}\left(n_{z}^{2}\right) \boldsymbol{n}_{\perp}^{A} \times[\hat{z} \times \boldsymbol{I}]+B_{\|}\left(n_{z}^{2}\right) \boldsymbol{n}_{\|}^{A} \times[\hat{z} \times \boldsymbol{I}] \\
& +C\left(n_{z}^{2}\right) \boldsymbol{n}_{\|}^{A} \times\left[\boldsymbol{n}_{\perp}^{A} \times[\hat{z} \times \boldsymbol{I}]\right],
\end{aligned}
$$

where all coefficients are generally even functions of the component $n_{z}=n_{z}^{A}=\cos \theta$. Note that the first two vector forms are of the same symmetry with respect to both reflections $\boldsymbol{n}_{\perp}^{A} \rightarrow-\boldsymbol{n}_{\perp}^{A}$ and $\boldsymbol{n}_{\|}^{A} \rightarrow-\boldsymbol{n}_{\|}^{A}$ :

Remarkably, our numerical data again correspond to a constant coefficient $A_{I}\left(n_{z}^{2}\right)=A_{I}$ in all regimes considered as was also the case for the symmetric model.

The spin orbit torque $\boldsymbol{T}^{A}$, which is obtained by substituting the result of Eq. (38) to Eq. (34), can be represented as the sum of only four vector forms

$$
\begin{aligned}
\boldsymbol{T}^{A}= & \eta\left(a_{I}\left(n_{z}^{2}\right) \boldsymbol{n}^{A} \times[\hat{z} \times \boldsymbol{j}]\right. \\
& +b_{\perp}\left(n_{z}^{2}\right) \boldsymbol{n}^{A} \times\left[\boldsymbol{n}_{\perp}^{A} \times[\hat{z} \times \boldsymbol{j}]\right] \\
& +b_{\|}\left(n_{z}^{2}\right) \boldsymbol{n}^{A} \times\left[\boldsymbol{n}_{\|}^{A} \times[\hat{z} \times \boldsymbol{j}]\right] \\
& \left.+c\left(n_{z}^{2}\right) \boldsymbol{n}^{A} \times\left[\boldsymbol{n}_{\|}^{A} \times\left[\boldsymbol{n}_{\perp}^{A} \times[\hat{z} \times j]\right]\right]\right),
\end{aligned}
$$

where we again introduce $\eta=J \mathcal{A} \Delta \lambda / e v h w^{2}, \boldsymbol{j}=\boldsymbol{I} / W$, and the dimensionless coefficients $a_{I}, b_{\perp}, b_{\|}$, and $c$ that all appear to be nontrivial functions of $n_{z}^{2}=\cos ^{2} \theta$ as shown in Fig. 11 . Note that the function $A_{I}^{\prime}\left(n_{z}^{2}\right)$ in Eq. (38) for spin density contributes to both $a_{I}$ and $c$ coefficients in Eq. (39) in the direct analogy to Eqs. (30) and (31).

Thus, the spin-orbit torque $\boldsymbol{T}^{A}$ is parameterized by four dimensionless functions. These functions are shown in Fig. 11 illustrating the main result of our numerical simulations for the asymmetric coupling model. In sharp contrast to the symmetric model we observe that the antidamping torques are no longer vanishing. We also find that $b_{\perp}\left(n_{z}^{2}\right) \approx b_{\|}\left(n_{z}^{2}\right)$ in the half-metal regime. The major fieldlike torque $\boldsymbol{n}^{A} \times[\hat{\boldsymbol{z}} \times \boldsymbol{j}]$, which originates in the Edelstein effect, acquires a weak dependence on $\theta$ due to the impact of the coefficient $A_{I}^{\prime}\left(n_{z}^{2}\right)$.

As is expected we observe that antidamping torques, which are proportional to $b_{\perp, \|}$, depend strongly on disorder concentration, while the fieldlike torques do not. Moreover, while antidamping torques are suppressed by disorder in the metal regime, the opposite is true in the half-metal regime.

Similarly to the symmetric model, the fieldlike torques are smaller in the metal regime than they are in half-metal regime. One can speculate, however, that anti-damping-like torques play a leading role in the metal regime of the asymmetric coupling model for sufficiently clean samples. We also see that antidamping torques reveal rather strong anisotropy. In the metal regime they take on maximal values for in-plane orientations of the vector $\boldsymbol{n}^{A}$.

It is also worth stressing that our results for asymmetric coupling model are the same for both ferromagnetic and antiferromagnetic order, since the spin-orbit torques are insensitive to the direction of the field $\boldsymbol{n}^{B}$.

As mentioned, one can expect the asymmetric model to capture also the physics of layered ferrimagnets such as

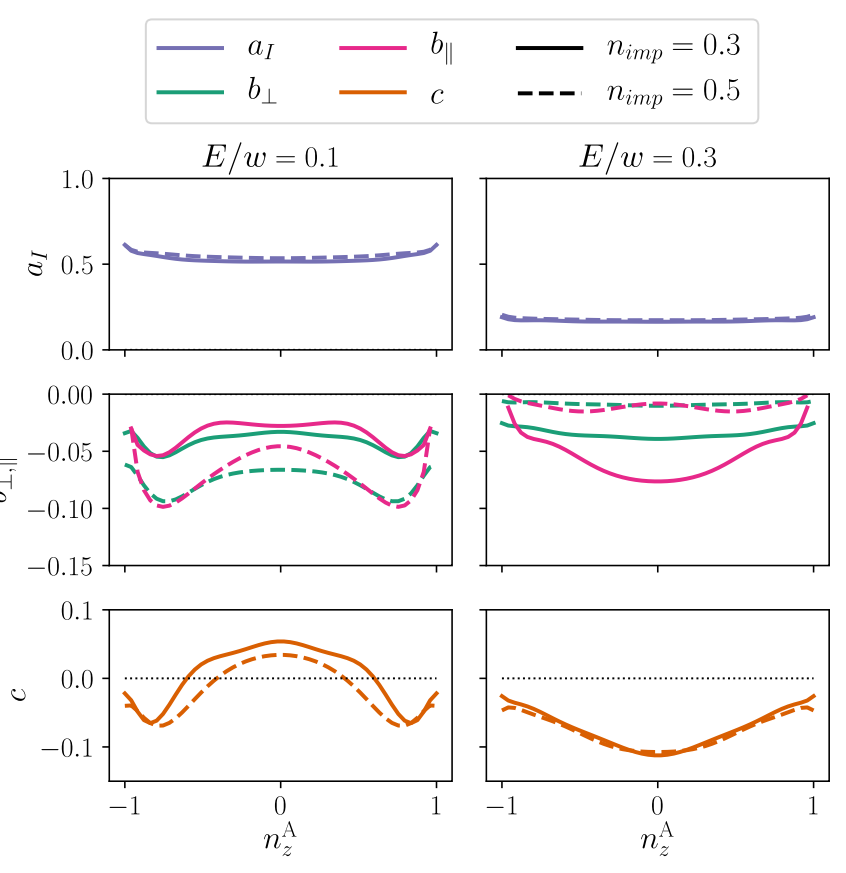

FIG. 11. Numerical results for the asymmetric coupling model that are expressed in the form of the angle-dependent coefficients $a_{I}$, $b_{\perp}, b_{\|}$, and $c$ that define the spin-orbit torque $\boldsymbol{T}^{A}$ in Eq. (39). The data are obtained by fitting the density data (partially represented in Fig. 10) using the ansatz of Eq. (38).

GdFeCo or Pt/GdFeCo [12,67]. The magnetization switching by means of spin-orbit torques in these materials has recently become a subject of intense studies [67-69]. In particular, the contribution from both fieldlike and anti-damping-like torques have been identified in GdFeCo films with perpendicular magnetocrystalline anisotropy in both transition-metal- and rareearth-metal-rich configurations $[68,69]$. These experimental results are, at least, qualitatively in line with our findings.

\section{ANTIFERROMAGNETIC DYNAMICS}

It is instructive to apply our results for the symmetric and asymmetric model to describe antiferromagnetic switching. As mentioned in the Introduction, both fieldlike staggered torque and anti-damping-like nonstaggered torque can be important for the Néel vector switching. In the symmetric model we only find fieldlike nonstaggered torques that cannot induce AFM switching for realistic values of the current intensity. In the asymmetric model we find both fieldlike and antidamping-like torques that act on a single sublattice. Although, strictly speaking, these do not entail a staggered torque, i.e., the Néel torque, one can nevertheless expect switching to occur.

In order to model AFM magnetization dynamics we adopt the equations of motion (20) for the unit vectors $\boldsymbol{n}^{A}$ and $\boldsymbol{n}^{B}$ with Gilbert damping terms included,

$$
\begin{aligned}
& \dot{\boldsymbol{n}}^{A}=\frac{J_{\mathrm{ex}}}{2 \hbar} \boldsymbol{n}^{B} \times \boldsymbol{n}^{A}+\frac{J \mathcal{A}}{\hbar} \boldsymbol{n}^{A} \times \boldsymbol{s}^{A}+\alpha \boldsymbol{n}^{A} \times \dot{\boldsymbol{n}}^{A}, \\
& \dot{\boldsymbol{n}}^{B}=\frac{J_{\mathrm{ex}}}{2 \hbar} \boldsymbol{n}^{A} \times \boldsymbol{n}^{B}+\frac{J \mathcal{A}}{\hbar} \boldsymbol{n}^{B} \times \boldsymbol{s}^{B}+\alpha \boldsymbol{n}^{B} \times \dot{\boldsymbol{n}}^{B},
\end{aligned}
$$



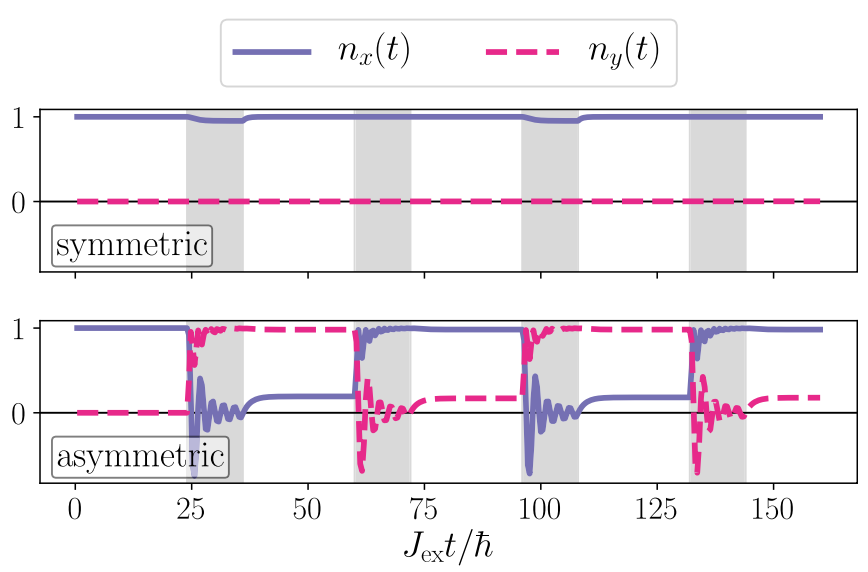

FIG. 12. Time evolution of the in-plane Néel vector $\boldsymbol{n}_{\|}(t)$ in the symmetric model (top panel) and asymmetric model (bottom panel) induced by a sequence of current pulses alternating between $-\hat{\boldsymbol{x}}$ and $\hat{\boldsymbol{y}}$ directions (gray areas). The results are obtained from Eqs. (40) for the parameters $\alpha=0.005, \hbar \eta j / J_{\mathrm{ex}}=0.05, E / w=0.3$, and $n_{\mathrm{imp}}=0.3$.

where we take $\alpha=0.005$ for the strength of the Gilbert damping.

In Fig. 12 we illustrate the AFM dynamics induced by short current pulses of the density $j=0.05 J_{\mathrm{ex}} / \eta \hbar$, which are applied in alternating directions $-\hat{\boldsymbol{x}}$ and $\hat{\boldsymbol{y}}$. The parameters of spin-orbit torques are derived for the Fermi energy $E=$ $0.3 w$ (in the metal regime) and for the impurity concentration $n_{\text {imp }}=0.3$ which corresponds to placing impurities on $30 \%$ of lattice sites.

The spin-orbit torques in the symmetric model are obtained by substituting $\boldsymbol{s}^{A}=\boldsymbol{s}^{B}=\delta \boldsymbol{s}_{+}$in Eqs. (40), where $\delta \boldsymbol{s}_{+} \propto$ $\hat{z} \times j$ is given by Eq. (26). The spin-orbit torques in the asymmetric model correspond to $\boldsymbol{s}^{B}=0$ and $\boldsymbol{s}^{A}$ taken from Eq. (38).

It is worth noting that the switching behavior in the asymmetric model (shown in the bottom panel of Fig. 12) is provided exclusively by the fieldlike torques [which are proportional to the $a_{I}$ and $c$ in Eq. (39)]. In contrast, antidamping torques, which are proportional to $b_{\perp, \|}$, do not play an important role in this behavior for the proposed choice of parameters. Nevertheless, if antidamping torques become essentially enhanced to overcome Gilbert damping, then they may lead to qualitatively different behavior. Indeed, for giant antidamping torques, the spin angular momentum in the asymmetric model is transferred from one sublattice to the another. Such a process, however, destroys the Néel order and fully magnetizes the sample during the current pulse. Once the electric current is switched off the Néel order is restored albeit in a different direction. This type of switching bears some resemblance to all-optical ultrafast magnetization switching that is observed, for example, in a ferrimagnet $\mathrm{GdFeCo}$ [70]. In the latter case the crystal also enters a transient fully magnetized state during a femtosecond optical pulse and relaxes to a state with opposite magnetization soon after the pulse. We note, however, that a current-induced switching of this type in our AFM model would require unrealistically large charge current intensities due to rather small magnitude of antidamping torques.

\section{CONCLUSIONS}

Motivated by recent experiments on the Néel vector switching we investigate microscopically the spin-orbit torques in an $s-d$-like model of a two-dimensional honeycomb antiferromagnet with Rashba spin-orbit coupling. We investigated the model with preserved and broken sublattice symmetry and distinguished metal and half-metal regimes for each of the model. Spin-orbit interaction in combination with onsite disorder potential and local exchange coupling between conduction and localized spins have been responsible for a microscopic mechanism of the angular-momentum relaxation. We find identically vanishing antidamping and Néel spin-orbit torques in the symmetric model in all regimes considered. As the result, the metal regime of the symmetric model is characterized by a particularly simple isotropic fieldlike spinorbit torque, while the half-metal regime is characterized by anisotropic spin-orbit torques of the fieldlike symmetry. Finite and anisotropic antidamping torques, which are essentially dissipative and, therefore, depend on disorder strength, are found in both metal and half-metal regimes of the asymmetric model. We also find nonequilibrium staggered polarization in the half-metal regime of the asymmetric model. This formally leads to a finite value of the Néel spin-orbit torque, that is, however, not a useful quantity in the asymmetric model. Overall, our results reveal the importance of two-dimensional electron momentum confinement for spin-orbit torque anisotropy. Largest values of spin-orbit torques are also associated with the half-metal regimes of conduction in both models.

\section{ACKNOWLEDGMENTS}

This research was supported by the JTC-FLAGERA Project GRANSPORT. D.Y. and M.T. acknowledge the support from the Russian Science Foundation Project No. 17-1201359. The work of D.Y. was also supported by the Swedish Research Council (Vetenskapsrådet, 2018-04383). A.M. and S.G. were supported by the King Abdullah University of Science and Technology (KAUST).
[1] S. Bader and S. Parkin, Annu. Rev. Condens. Matter Phys. 1, 71 (2010).

[2] J. Sinova and I. Žutić, Nat. Mater. 11, 368 (2012).

[3] S. Bhatti, R. Sbiaa, A. Hirohata, H. Ohno, S. Fukami, and S. N. Piramanayagam, Mater. Today 20, 530 (2017).
[4] A. D. Kent and D. C. Worledge, Nat. Nanotechnol. 10, 187 (2015).

[5] N. Sato, F. Xue, R. M. White, C. Bi, and S. X. Wang, Nat. Electron. 1, 508 (2018).

[6] A. H. MacDonald and M. Tsoi, Philos. Trans. R. Soc. A 369, 3098 (2011). 
[7] E. V. Gomonay and V. M. Loktev, Low Temp. Phys. 40, 17 (2014).

[8] P. Wadley, B. Howells, J. Železný, C. Andrews, V. Hills, R. P. Campion, V. Novák, K. Olejník, F. Maccherozzi, S. S. Dhesi et al., Science 351, 587 (2016).

[9] T. Jungwirth, X. Marti, P. Wadley, and J. Wunderlich, Nat. Nanotechnol. 11, 231 (2016).

[10] V. Baltz, A. Manchon, M. Tsoi, T. Moriyama, T. Ono, and Y. Tserkovnyak, Rev. Mod. Phys. 90, 015005 (2018).

[11] T. Jungwirth, J. Sinova, A. Manchon, X. Marti, J. Wunderlich, and C. Felser, Nat. Phys. 14, 200 (2018).

[12] M. B. Jungfleisch, W. Zhang, and A. Hoffmann, Phys. Lett. A 382, 865 (2018).

[13] O. Gomonay, T. Jungwirth, and J. Sinova, Phys. Rev. Lett. 117, 017202 (2016).

[14] K. Olejník, T. Seifert, Z. Kašpar, V. Novák, P. Wadley, R. P. Campion, M. Baumgartner, P. Gambardella, P. Němec, J. Wunderlich et al., Sci. Adv. 4, eaar3566 (2018).

[15] I. Fina and X. Marti, IEEE Trans. Magn. 53, 1 (2017).

[16] A. Brataas, A. D. Kent, and H. Ohno, Nat. Mater. 11, 372 (2012).

[17] K. M. D. Hals and A. Brataas, Phys. Rev. B 88, 085423 (2013).

[18] J. Železný, H. Gao, K. Výborný, J. Zemen, J. Mašek, A. Manchon, J. Wunderlich, J. Sinova, and T. Jungwirth, Phys. Rev. Lett. 113, 157201 (2014).

[19] F. Freimuth, S. Blügel, and Y. Mokrousov, Phys. Rev. B 90, 174423 (2014)

[20] S. Ghosh and A. Manchon, Phys. Rev. B 95, 035422 (2017).

[21] L. Šmejkal, J. Železný, J. Sinova, and T. Jungwirth, Phys. Rev. Lett. 118, 106402 (2017).

[22] J. Železný, P. Wadley, K. Olejník, A. Hoffmann, and H. Ohno, Nat. Phys. 14, 220 (2018).

[23] X. F. Zhou, J. Zhang, F. Li, X. Z. Chen, G. Y. Shi, Y. Z. Tan, Y. D. Gu, M. S. Saleem, H. Q. Wu, F. Pan et al., Phys. Rev. Appl. 9, 054028 (2018).

[24] A. Manchon, J. Zelezný, I. M. Miron, T. Jungwirth, J. Sinova, A. Thiaville, K. Garello, and P. Gambardella, Rev. Mod. Phys. 91, 035004 (2019).

[25] T. Moriyama, W. Zhou, T. Seki, K. Takanashi, and T. Ono, Phys. Rev. Lett. 121, 167202 (2018).

[26] Y. Li, K. W. Edmonds, X. Liu, H. Zheng, and K. Wang, Adv. Quant. Technol. 2, 1800052 (2019).

[27] X. Chen, X. Zhou, R. Cheng, C. Song, J. Zhang, Y. Wu, Y. Ba, H. Li, Y. Sun, Y. You et al., Nat. Mater. 18, 931 (2019).

[28] J. Zhou, X. Wang, Y. Liu, J. Yu, H. Fu, L. Liu, S. Chen, J. Deng, W. Lin, X. Shu et al., Sci. Adv. 5, eaau6696 (2019).

[29] X. F. Zhou, X. Z. Chen, J. Zhang, F. Li, G. Y. Shi, Y. M. Sun, M. S. Saleem, Y. F. You, F. Pan, and C. Song, Phys. Rev. Appl. 11, 054030 (2019).

[30] S. Y. Bodnar, L. Šmejkal, I. Turek, T. Jungwirth, O. Gomonay, J. Sinova, A. A. Sapozhnik, H.-J. Elmers, M. Kläui, and M. Jourdan, Nat. Commun. 9, 348 (2018).

[31] V. Saidl, P. Němec, P. Wadley, V. Hills, R. P. Campion, V. Novák, K. W. Edmonds, F. Maccherozzi, S. S. Dhesi, B. L. Gallagher et al., Nat. Photon. 11, 91 (2017).

[32] V. M. T. S. Barthem, C. V. Colin, H. Mayaffre, M.-H. Julien, and D. Givord, Nat. Commun. 4, 1 (2013).

[33] M. Jourdan, H. Bräuning, A. Sapozhnik, H.-J. Elmers, H. Zabel, and M. Kläui, J. Phys. D: Appl. Phys. 48, 385001 (2015).
[34] N. Bhattacharjee, A. A. Sapozhnik, S. Y. Bodnar, V. Y. Grigorev, S. Y. Agustsson, J. Cao, D. Dominko, M. Obergfell, O. Gomonay, J. Sinova et al., Phys. Rev. Lett. 120, 237201 (2018).

[35] S. K. Pandey, P. Mahadevan, and D. D. Sarma, Europhys. Lett. 117, 57003 (2017).

[36] V. Tshitoyan, C. Ciccarelli, A. P. Mihai, M. Ali, A. C. Irvine, T. A. Moore, T. Jungwirth, and A. J. Ferguson, Phys. Rev. B 92, 214406 (2015).

[37] J. Železný, H. Gao, A. Manchon, F. Freimuth, Y. Mokrousov, J. Zemen, J. Mašek, J. Sinova, and T. Jungwirth, Phys. Rev. B 95, 014403 (2017).

[38] E. L. Fjærbu, N. Rohling, and A. Brataas, Phys. Rev. B 95, 144408 (2017).

[39] R. Cheng, D. Xiao, and A. Brataas, Phys. Rev. Lett. 116, 207603 (2016).

[40] R. Khymyn, I. Lisenkov, V. Tiberkevich, B. A. Ivanov, and A. Slavin, Sci. Rep. 7, 43705 (2017).

[41] A. Manchon, J. Phys.: Condens. Matter 29, 104002 (2017).

[42] S. Ghosh and A. Manchon, Phys. Rev. B 100, 014412 (2019).

[43] H. Watanabe and Y. Yanase, Phys. Rev. B 98, 220412(R) (2018).

[44] K. M. D. Hals, Y. Tserkovnyak, and A. Brataas, Phys. Rev. Lett. 106, 107206 (2011).

[45] J. Barker and O. A. Tretiakov, Phys. Rev. Lett. 116, 147203 (2016).

[46] X. Zhang, Y. Zhou, and M. Ezawa, Sci. Rep. 6, 1 (2016).

[47] J. C. Slonczewski, J. Magn. Magn. Mater. 159, L1 (1996).

[48] T. Shiino, S.-H. Oh, P. M. Haney, S.-W. Lee, G. Go, B.-G. Park, and K.-J. Lee, Phys. Rev. Lett. 117, 087203 (2016).

[49] C. A. Akosa, O. A. Tretiakov, G. Tatara, and A. Manchon, Phys. Rev. Lett. 121, 097204 (2018).

[50] R. Tomasello, V. Puliafito, E. Martinez, A. Manchon, M. Ricci, M. Carpentieri, and G. Finocchio, J. Phys. D: Appl. Phys. 50, 325302 (2017).

[51] C. W. Groth, M. Wimmer, A. R. Akhmerov, and X. Waintal, New J. Phys. 16, 063065 (2014).

[52] C. L. Kane and E. J. Mele, Phys. Rev. Lett. 95, 146802 (2005).

[53] Z. Qiao, H. Jiang, X. Li, Y. Yao, and Q. Niu, Phys. Rev. B 85, 115439 (2012).

[54] M. Ezawa, Phys. Rev. Lett. 110, 026603 (2013).

[55] I. A. Ado, O. A. Tretiakov, and M. Titov, Phys. Rev. B 95, 094401 (2017).

[56] I. A. Ado, P. M. Ostrovsky, and M. Titov, arXiv:1907.02041 [cond-mat] (2019).

[57] S. Datta, Electronic Transport in Mesoscopic Systems, Cambridge Studies in Semiconductor Physics and Microelectronic Engineering (Cambridge University Press, Cambridge, 1995).

[58] E. van der Bijl and R. A. Duine, Phys. Rev. B 86, 094406 (2012).

[59] K. Garello, I. M. Miron, C. O. Avci, F. Freimuth, Y. Mokrousov, S. Blügel, S. Auffret, O. Boulle, G. Gaudin, and P. Gambardella, Nat. Nanotechnol. 8, 587 (2013).

[60] V. M. Edelstein, Solid State Commun. 73, 233 (1990).

[61] A. Manchon and S. Zhang, Phys. Rev. B 78, 212405 (2008).

[62] I. Garate and A. H. MacDonald, Phys. Rev. B 80, 134403 (2009).

[63] H. van Leuken and R. A. de Groot, Phys. Rev. Lett. 74, 1171 (1995). 
[64] S.-J. Gong, C. Gong, Y.-Y. Sun, W.-Y. Tong, C.-G. Duan, J.H. Chu, and X. Zhang, Proc. Natl. Acad. Sci. USA 115, 8511 (2018).

[65] H. Kurt, K. Rode, P. Stamenov, M. Venkatesan, Y.-C. Lau, E. Fonda, and J. M. D. Coey, Phys. Rev. Lett. 112, 027201 (2014).

[66] D. Betto, K. Rode, N. Thiyagarajah, Y.-C. Lau, K. Borisov, G. Atcheson, M. Žic, T. Archer, P. Stamenov, and J. M. D. Coey, AIP Adv. 6, 055601 (2016).
[67] J. Kim, D. Lee, K.-J. Lee, B.-K. Ju, H. C. Koo, B.-C. Min, and O. Lee, Sci. Rep. 8, 1 (2018).

[68] N. Roschewsky, T. Matsumura, S. Cheema, F. Hellman, T. Kato, S. Iwata, and S. Salahuddin, Appl. Phys. Lett. 109, 112403 (2016).

[69] N. Roschewsky, C.-H. Lambert, and S. Salahuddin, Phys. Rev. B 96, 064406 (2017).

[70] A. Kirilyuk, A. V. Kimel, and T. Rasing, Rev. Mod. Phys. 82, 2731 (2010). 